\title{
INFLUENCE OF KIND OF WEFT YARN ON PROPERTIES OF THE SEERSUCKER WOVEN FABRICS
}

\author{
Małgorzata Matusiak ${ }^{1}$, Łukasz Frącczak ${ }^{2}$ \\ IInstitute of Architecture of Textiles, Lodz University of Technology; malgorzata.matusiak@p.lodz.pl \\ ${ }^{2}$ Faculty of Mechanical Engineering Institute of Machine Tools and Production Engineering, Lodz University of Technology, \\ Lodz, Poland; lukasz.fracczak@p.lodz.pl
}

\begin{abstract}
:
Seersucker woven fabrics create a unique 3D woven structure. Such 3D structure is usually received on loom by an application of two warps of different tension. However, a kind of the weft yarn also significantly influences the structure and properties of the seersucker woven fabric. The paper presents an investigation of the seersucker fabrics made of the same set of warps and different weft yarns. The mechanical properties of the investigated fabrics were measured by means of the standardized testing methods. The structure of the fabric was assessed using the 3D laser scanning.
\end{abstract}

\section{Keywords:}

Woven fabrics, seersucker fabrics, breaking force, bending stiffness, total height of the profile, laser scanning, seersucker effect

\section{Introduction}

Seersucker fabrics create a unique 3D woven structure. Such 3D structure is usually received on loom by an application of two warps of different tension [1, 2]. A typical seersucker structure is characterized by an occurring the puckered strips in the warp direction. The word "seersucker" came into English from Persian and originates from the words "Sheer" and "Shakar," which mean "milk and sugar," probably from the similarity of smooth and rough stripes along the fabric to the smooth texture of milk and the lumpy texture of sugar [3]. The seersucker fabrics are also called goffered fabrics.

To make the seersucker woven fabric, two loom beams are needed: one beam carries warp yarns for the flat (basic) stripes and the other carries warp yarns for the puckered stripes. During weaving, adjustments are made to make the puckered stripe warp yarns feed forward faster than the flat stripe warp yarns. This results in different tension of warp yarns, and following it, a localized buckling of the fabric in the areas of the fast-feeding yarns is formed [2, 4]. This makes the pucker in the wrinkled stripes in warp direction. Figure 1 presents an example of the seersucker fabric made of two warps of different tension and one kind of weft yarn. Sometimes such a structure is called $2 D+$ woven fabric.

Owing to the use of two beams, the typical seersucker woven fabrics cannot be manufactured on conventional looms with single warp beam. Maqsooda et al. [5] developed the seersucker woven fabrics produced on conventional looms using a single warp beam. For this purpose, two types of weft yarn, $100 \%$ cotton yarns and blended yarns of cotton and elastane $(95.67 \%$ cotton and $4.33 \%$ Lycra), were used in groups. The weft yarns were distributed in two stripes: one is having cotton threads and the other having both cotton and core spun yarns. It was stated that the amount of Lycra changes the visual appearance or puckering effect and the properties of fabric: elasticity and stiffness [5].

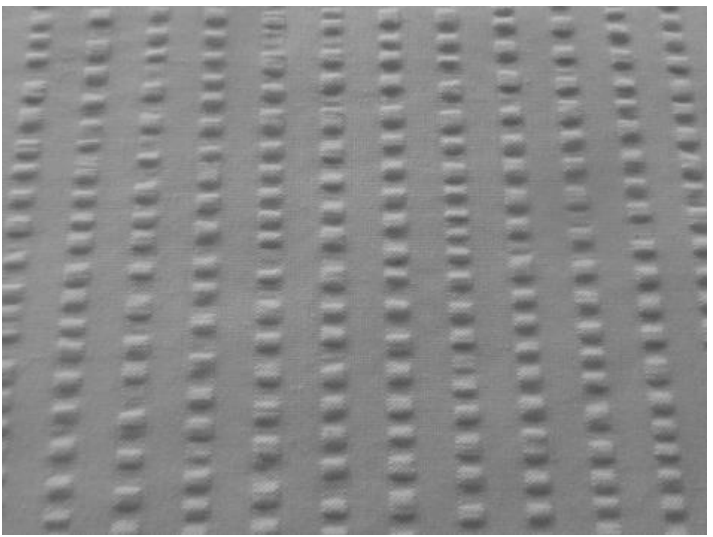

Figure 1. An example of the seersucker woven fabric

The elastane yarn was also applied in 3D woven fabrics by Matusiak et al. [6]. They designed the seersucker woven structure by an appropriate combination of two warp yarns of different tensions (typical two beam seersucker effect) and two weft yarns of different elasticity. In this case, the puckering effect was visible in both warp and weft directions (Figure 2).

Owing to the puckered (goffered) structure and special surface properties of the developed 3D woven fabric, it was characterized by its stiffness, static and dynamic friction and thermal resistance. Thanks to mentioned features the fabric ensures the mechanical micro-massage and stimulation of the blood circulation. The developed fabric was applied in anticellulite trousers [6]. 


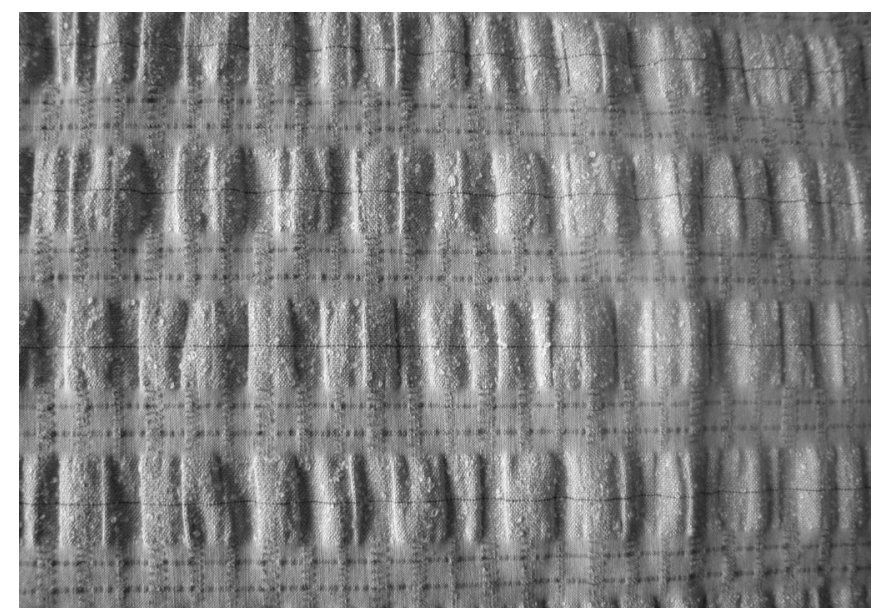

Figure 2. 3D woven fabric of randomly distributed convex places [6]

Ashraf et al. described the knitted technique of the seersucker fabric formation [7]. They manufactured the knitted seersucker fabric on single cylinder weft knitting machine using plain jersey structure. In order to obtain the puckering effect, two different kinds of yarn were applied: $100 \%$ cotton yarn and core spun yarn with cotton fibers in the sheath and elastane filament in the core. Prepared in such a way, the knitted fabric was characterized by the strips in course direction.

The wrinkled-striped structure of the seersucker fabrics causes that the fabrics are carefree: they do not need to be pressed. It is an important advantage of such kind of fabrics, especially for apparel ensuring a utility comfort. Some researchers $[4,5,7,8]$ considered that the seersucker fabrics have good comfort properties because of the puckered structure. The puckered effect generates air spaces between body and the fabric, keeping the wearer cool in hot conditions as the puckered area holds the fabric away from the skin while usage. It facilitates air circulation. However, the thesis is not obvious and should be discussed, especially in the case of fabrics containing elastomeric yarns. An application of elastomeric yarn in weft causes that the fabric fits closely to user's body. It can impede heat and air exchange between the body and surroundings.

The application of two warp sets in the seersucker woven fabric influences both the appearance of the fabric and its properties. A kind of warp yarns, number of threads of basic and goffering (puckering) warps, as well as the relation between the tension of the basic and goffering warps during weaving play a crucial role in shaping the properties of the seersucker fabrics. However, a kind of the weft yarn also significantly influences the structure and properties of the fabrics.

The seersucker effect is one of the most important factor influencing the appearance, properties, and performance of the seersucker fabrics. Until now, the seersucker effect has been assessed quantitatively by measuring the percentage of shrinkage of the shrunk parts. Ghahraman et al. presented two algorithms, namely, spectral density function and angular power spectrum function, capable of assessing the three-dimensional seersucker effect qualitatively [9]. Matusiak and Fracczak [1, 10] applied the 3D laser scanner for measuring the 3D woven fabrics of small-scale evenly distributed three-dimensionality. They calculated a profile parameter - the total height of the profile $W_{z}[11,12]$ for lines extracted from the fabrics' scans. The lines represented the profile of the fabrics in both warp and weft directions. Performed investigations confirmed that the $\mathrm{W}_{\mathrm{z}}$ parameter can be considered as a measure of topography of the $3 D$ woven fabrics $[1,10]$.

The aim of the presented work is to analyze the structure and mechanical properties of the seersucker woven fabrics as well as the influence of the kind of weft yarn on chosen properties of the fabrics.

\section{Experimental}

The paper presents the investigations of the typical seersucker fabrics made of the same set of warps and different weft yarns. Investigated fabrics were manufactured on the basis of two warps: warp I, basic; and warp II, goffering (puckering), both made of $20 \times 2$ tex cotton yarn. Five kinds of yarn were applied in weft. They were:

- $20 \times 2$ tex cotton yarn,

- $15 \times 2$ tex CO50/PES50,

- $15.6 \times 2$ tex PES 156/94/2 multifilament yarn,

- $12 \times 2$ tex PES 88/SeeCell Active 12,

- 37 tex PU 57/PES 43 elastomeric yarn.

The seersucker fabrics were produced on a loom with two beams. First, the trials were performed in order to establish an appropriate weft density. It was done because of the fact that the applied weft yarns differed between each other not only in the range of fiber composition but also from the point of view of yarn linear density and diameter. For each fabric variant, the weft density was established in such a way to obtain correct, stable seersucker effect. As a result, in the fabrics No. 3 and 4 , a weft density was higher than in fabrics No. 1, 2 and 5. Next, the fabrics were finished by the same way including washing, drying, and stabilization processes.

The basic structural parameters of the investigated fabrics are presented in Table 1.

The manufactured seersucker fabrics were measured in the range of the basic structural and mechanical parameters. Measurements of the basic structural parameters were performed using standardized testing methods. Results of the measurement are presented in Table 2.

The results confirmed that the application of different kinds of yarn in weft significantly influences the structural and mechanical properties of the investigated fabrics. The differences were stated in the range of all measured parameters. Especially, the sample No. 5 has quite different structure than other analyzed fabrics. It is due to the application of the elastomeric yarn in weft. It caused that the structure of the fabric No. 5 is very tight in weft direction. Owing to the tight structure, the fabric No. 5 is characterized by the highest mass per square meter, warp density, and take-up of the warp I. 
Table 1. Characteristic of the manufactured seersucker woven fabrics

\begin{tabular}{|l|c|c|c|c|c|c|}
\hline \multirow{2}{*}{ Parameter } & \multirow{2}{*}{ Unit } & \multicolumn{4}{|c|}{ Value } \\
\cline { 3 - 7 } & & Sample 1 & Sample 2 & Sample 3 & Sample 4 & Sample 5 \\
\hline Warp I & - & $20 \times 2$ tex CO & $20 \times 2$ tex CO & $20 \times 2$ tex CO & $20 \times 2$ tex CO & $20 \times 2$ tex CO \\
\hline Warp II & - & $20 \times 2$ tex CO & $20 \times 2$ tex CO & $20 \times 2$ tex CO & $20 \times 2$ tex CO & $20 \times 2$ tex CO \\
\hline Weft & - & $20 \times 2$ tex CO & $\begin{array}{c}15 \times 2 \text { tex CO50/ } \\
\text { PES50 }\end{array}$ & $\begin{array}{c}15.6 \times 2 \text { PES } \\
156 / 94 / 2\end{array}$ & $\begin{array}{c}12 \times 2 \text { tex } \\
\text { PES } 88 /\end{array}$ & $\begin{array}{c}37 \text { tex } \\
\text { PeeCell } 12\end{array}$ \\
\hline $\begin{array}{l}\text { Nominal weft } \\
\text { density }\end{array}$ & $\mathrm{dm}^{-1}$ & 200 & 230 & 280 & 260 & 200 \\
\hline Weave - warp I & - & plain & plain & plain & plain & plain \\
\hline Weave - warp II & - & rep 2/2 & rep 2/2 & rep 2/2 & rep 2/2 & rep 2/2 \\
\hline
\end{tabular}

Table 2. Basic parameters of the investigated seersucker woven fabrics

\begin{tabular}{|c|c|c|c|c|c|c|}
\hline \multirow{2}{*}{ Parameter } & \multirow{2}{*}{ Unit } & \multicolumn{5}{|c|}{ Value } \\
\hline & & Sample 1 & Sample 2 & Sample 3 & Sample 4 & Sample 5 \\
\hline $\begin{array}{l}\text { Mass per square } \\
\text { meter }\end{array}$ & $\mathrm{gm}^{-2}$ & 241 & 224 & 201 & 208 & 384 \\
\hline Warp density & $\mathrm{dm}^{-1}$ & 278 & 281 & 284 & 274 & 393 \\
\hline Weft density & $\mathrm{dm}^{-1}$ & 221 & 237 & 290 & 281 & 240 \\
\hline Warp I take-up & $\%$ & 5.12 & 3.32 & 1.85 & 5.05 & 16.9 \\
\hline Warp II take-up & $\%$ & 70.6 & 54.5 & 60.0 & 62.9 & 64.2 \\
\hline Weft take up & $\%$ & 13.7 & 13.0 & 15.9 & 10.3 & 67.8 \\
\hline Thickness & $\mathrm{mm}$ & 1.64 & 1.41 & 1.31 & 1.49 & 1.58 \\
\hline
\end{tabular}

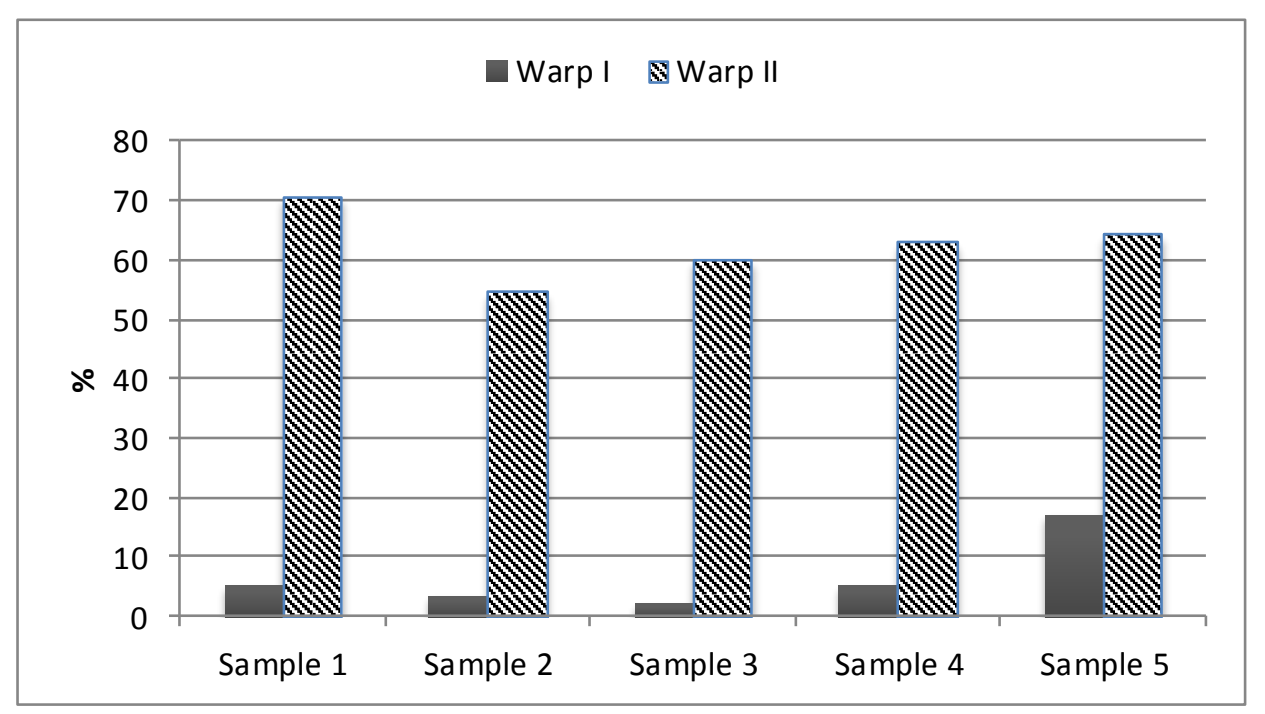

Figure 3. Warp take-up of the investigated seersucker fabrics

All fabrics are characterized by very high take-up of the warp II, much higher than the take-up of the warp I (Figure 3). It is a typical feature of the seersucker fabrics. The difference between the take-up of the basic and goffering warps can also be considered as a measure of the seersucker effect.
The highest difference between the take-up of the basic and goffering warp occurs in fabric No. 1 made of the $20 \times 2$ tex cotton yarn in weft; the lowest is in the fabric No. 5 with the elastomeric weft yarn (Figure 4). However, the sample No. 5 is characterized by the highest take-up of the warp I. It causes 


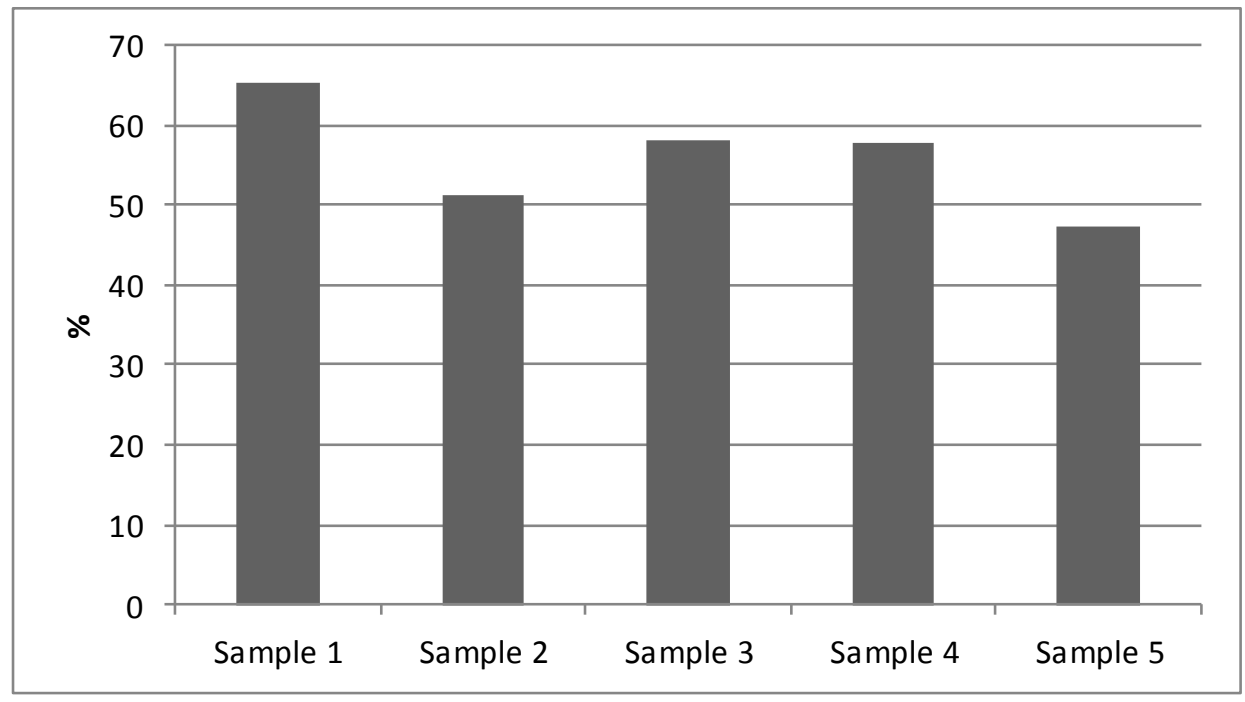

Figure 4. The difference between the take-up of the basic and goffering warps

that although the take-up of the goffering warp is high, the difference between both warps basic and goffering in the range of the take-up is low.

The surface topography [11] of the investigated seersucker fabrics was assessed using the 3D laser scanning. The testbench consisted of the CimCore Romer Infinity 2 measuring arm with the Perceptron's Contour Probe laser head was used in the measurement. Prepared scans of the fabric surface were exported to the format appropriate to use by the CAD software. Next, the scans were cut in both directions, warp and weft, in order to obtain the fabric sections and to extract the lines representing the profile of the fabric in the place of cutting. The series of parallel lines spaced each $1 \mathrm{~mm}$ were generated in such a way to cover at least the full pattern repeat defined along the warp and the weft (Figure 5).

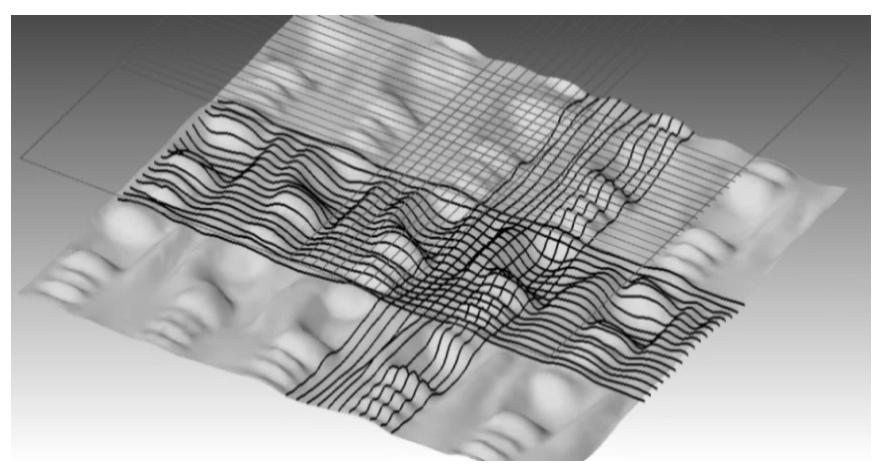

Figure 5. A series of lines created in both warp and weft directions

Each projection of the line was divided into five sections of $10 \mathrm{~mm}$ in length. Next, the total height of the profile $W_{z}[12]$ was measured for each section. The average values of the total height of the profile in the warp and weft directions were calculated as a final result. Figure 6 presents an example of the results - the average values of the total height of the profile $W_{z}$ for lines created in warp direction for sample No. 1.

The lines created in warp direction can be divided into two groups: the lines along the flat strip and lines along the puckered strip. The lines of higher values of the $W_{z}$ parameter represent the seersucker strip, whereas the lines of lower values of the $W_{z}$ parameter represent the basic (flat) strip. The results presented in Figure 6 clearly show the seersucker effect. However, in our opinion, the difference between the maximal and minimal value of the $W_{z}$ parameter can be a measure, which quantifies the seersucker effect better than the values of the total height of the profile of particular lines. We can see that (with exception of sample No. 2) the difference between the maximal and minimal value of the $W_{z}$ parameter in the warp direction of investigated fabrics (Figure 7) shows the same tendency than the difference between the take-up of the basic and goffering warps (Figure 4). In both the cases, the highest value was stated for the sample No. 1, the lowest is for the sample No. 5 .

From the lines extracted from the scans of fabrics, we can also read the width of the puckered and flat strips. It is due to the fact that the lines were created each $1 \mathrm{~mm}$. It means that one line corresponds to about $1 \mathrm{~mm}$. As we can see from Figure 6 , in the case of sample No. 1, the number of lines corresponding to the puckered strip is 6 , whereas the number of lines expressing the flat strip is 7 . The lines No. 8 and 13 can be considered as the border lines of the puckered strip.

In order to compare different seersucker fabrics, we can express the width of strips in number of lines relating to puckered and flat strips (Figure 8).

We can see that the biggest number of lines corresponding to the puckered strip occurred for samples No. 1 and 3. It means that in these fabrics, the puckered strips are the widest. The sample No. 5 is characterized by the lowest number of lines corresponding to the flat strip. At the same time, the sample No. 5 is characterized by the narrowest flat strips. It is due to the application of the elastomeric yarn in weft, what significantly influenced the appearance and structure of the fabric No. 5.

Table 3 presents the mechanical properties of the investigated seersucker fabrics. Breaking force, elongation at break, and 


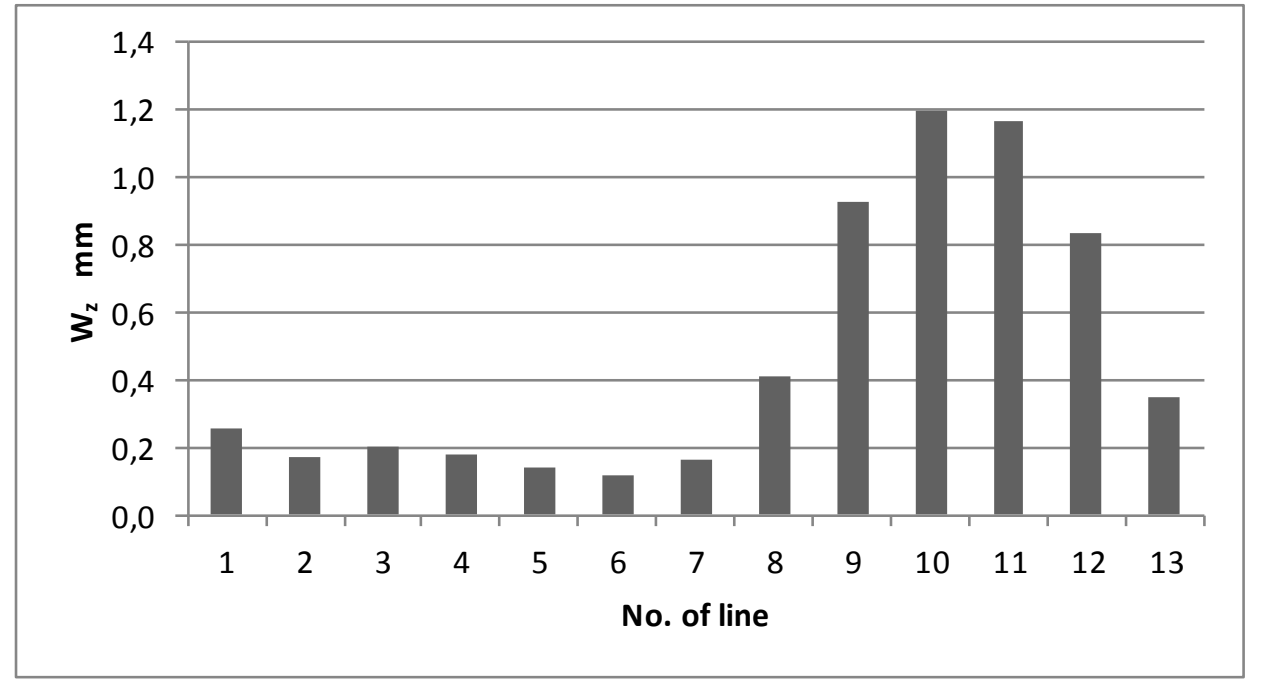

Figure 6. The average values of the total height of the profile $\left(\mathrm{W}_{\mathrm{z}}\right)$ for the lines created in warp direction for the sample No. 1

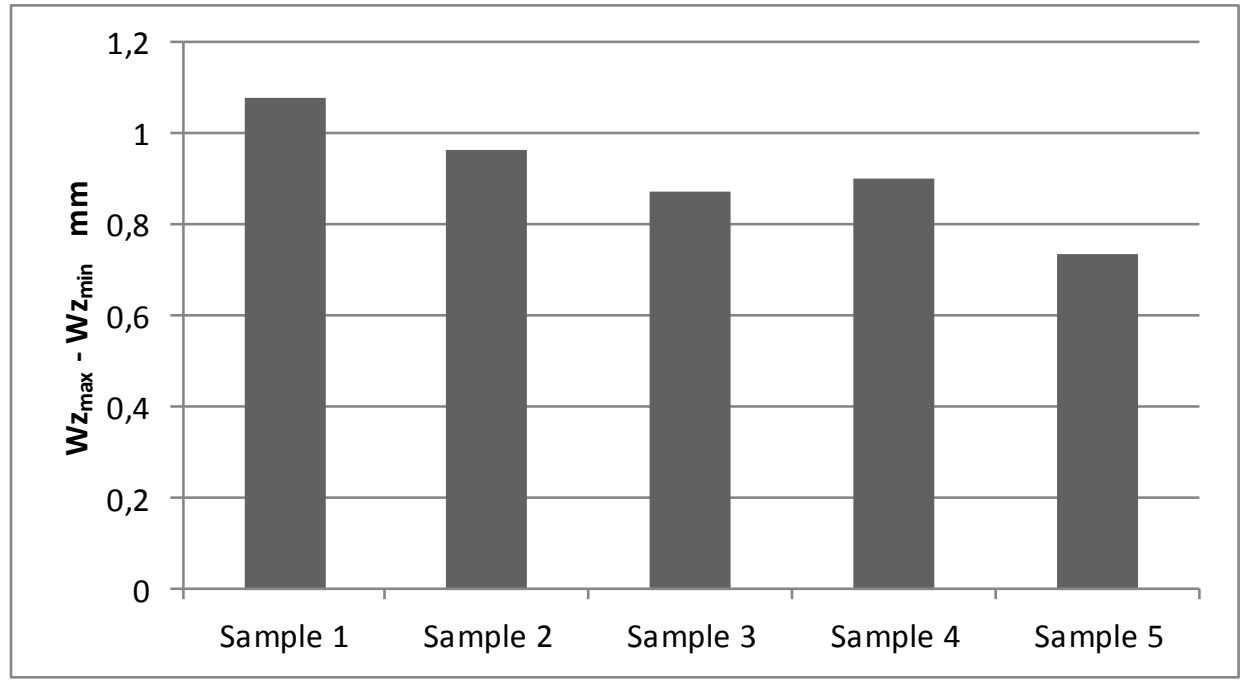

Figure 7. The difference between the maximal and minimal value of the $W_{z}$ parameter in the warp direction of investigated fabrics

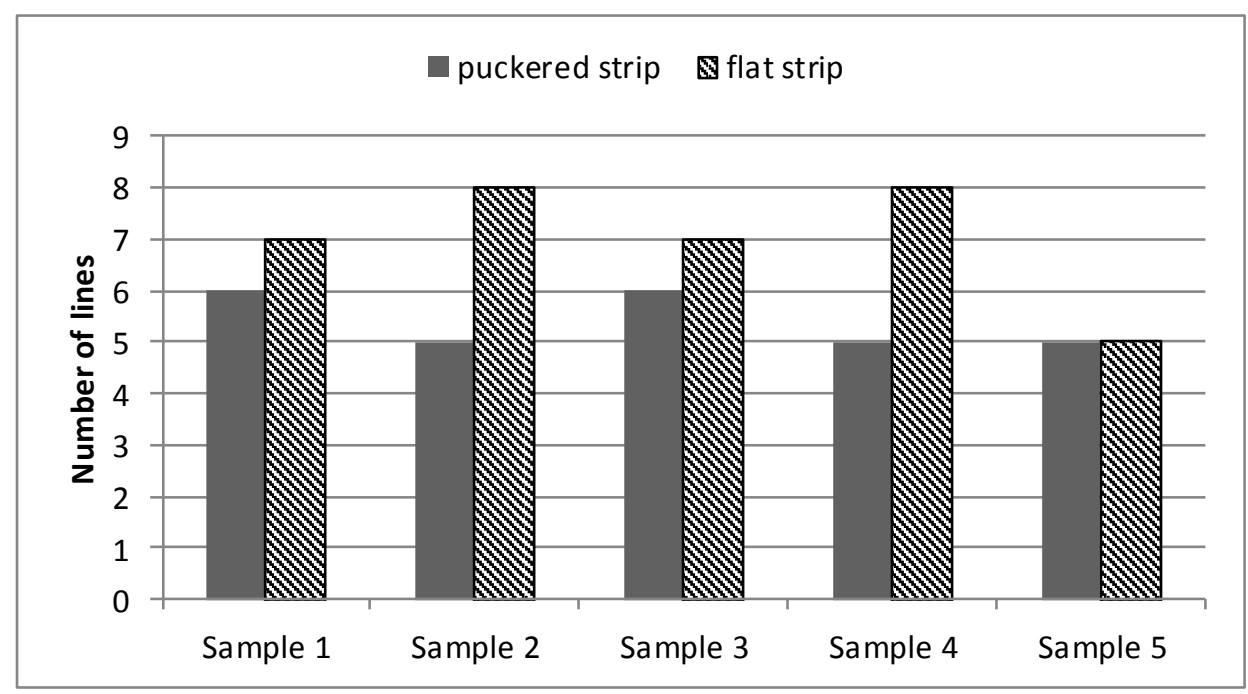

Figure 8. Number of lines corresponding to the puckered and flat strips 
Table 3. Mechanical properties of the investigated seersucker woven fabrics

\begin{tabular}{|l|c|c|c|c|c|c|}
\hline \multirow{2}{*}{ Parameter } & \multirow{2}{*}{ Unit } & \multicolumn{5}{|c|}{ Value } \\
\cline { 3 - 7 } & & Sample 1 & Sample 2 & Sample 3 & Sample 4 & Sample 5 \\
\hline Breaking force -warp & $\mathrm{N}$ & 425.0 & 485.5 & 467.4 & 434.0 & 629.0 \\
\hline Breaking force - weft & $\mathrm{N}$ & 663.0 & 551.0 & 660.0 & 684.0 & 493.0 \\
\hline Elongation at break - warp & $\%$ & 12.40 & 10.77 & 10.37 & 12.78 & 27.00 \\
\hline Elongation at break - weft & $\%$ & 21.23 & 25.10 & 36.94 & 27.88 & 148.9 \\
\hline Stiffness - warp direction & $\mathrm{mNm}$ & 0.041 & 0.047 & 0.042 & 0.036 & 0.280 \\
\hline Stiffness - weft direction & $\mathrm{mNm}$ & 0.046 & 0.035 & 0.016 & 0.027 & 0.110 \\
\hline Stiffness - total & $\mathrm{mNm}$ & 0.043 & 0.041 & 0.026 & 0.031 & 0.174 \\
\hline
\end{tabular}

bending stiffness were measured using standardized testing methods.

In majority of the cases (Figure 9), the breaking force in warp direction is lower than that in weft direction. It is unusual situation, because in majority of the woven fabrics, relation is opposite. However, it is justified by the fact that in seersucker fabrics, the load in warp is carried by the part of warp threads belonging to one warp set creating the flat strips. The warp threads belonging to the goffered strips are puckered and under the stretching force they are straightened. In the case of the sample No. 5, the breaking force in warp direction is much higher than that in weft direction. Moreover, the breaking force in the warp direction of the sample 5 is significantly higher than that for the rest of investigated fabrics. It is due to the fact that the warp density of the sample 5 is more than 1.5 times higher than the warp density of the samples No. $1-4$. It means that the application of the elastomeric yarn in weft of the sample No. 5 caused the tightening of the fabric structure in weft direction, and at the same time, it caused a higher number of warp threads carrying the breaking force in warp direction what resulted in higher tensile tenacity of the sample No. 5 in comparison to other investigated fabrics.
The application of the elastomeric yarn in weft direction of the sample No. 5 also influenced its elongation at break (Figure 10). We can see that the elongation at break in weft direction of the sample No. 5 is about $150 \%$, and it is more than 4 times higher than the elongation at break in weft direction of the rest of investigated samples. In warp direction, the elongation at break of the sample No. 5 is twice higher than that for the samples No. 1 - 4. We can also see that for all investigated fabrics, the elongation at break in warp direction is much (sometimes twice) lower than in weft direction. It is due to the fact that in weft direction first the straightening the structure takes place and next the breaking the fabrics.

We can also observe significant differences in bending stiffness of fabrics (Figure 11). The highest bending stiffness in both directions, warp and weft, as well as the total bending stiffness occurred for the sample No. 5 . It is caused by the significantly higher warp density of the sample 5 in comparison to the other samples (Table 2). Analyzing the results of fabrics No. 1-4, we can see that the bending stiffness in warp direction is at similar level. Significant differences occur in the range of the bending stiffness in weft direction. The lowest bending stiffness in weft direction was stated for sample No. 3 in which the weft is created by the multifilament yarn $15.6 \times 2$ PES 156/94/2. This

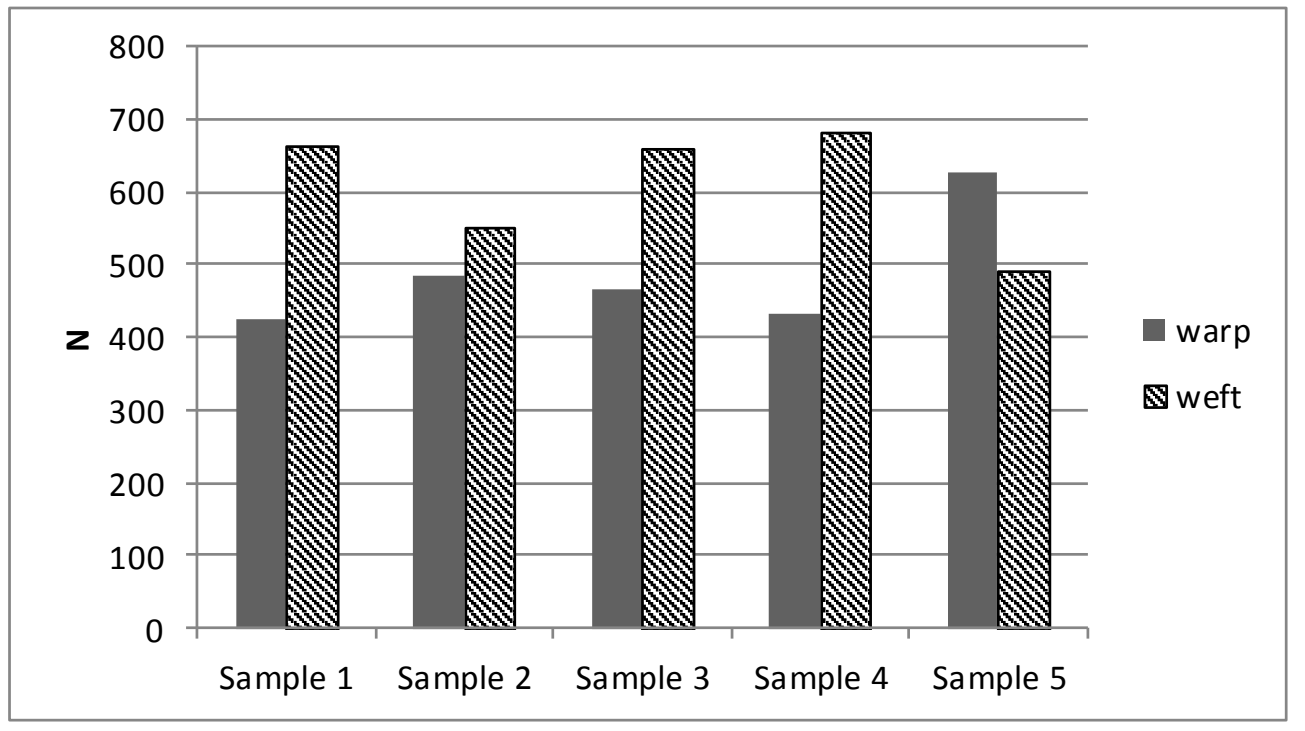

Figure 9. Breaking force of investigated seersucker fabrics 


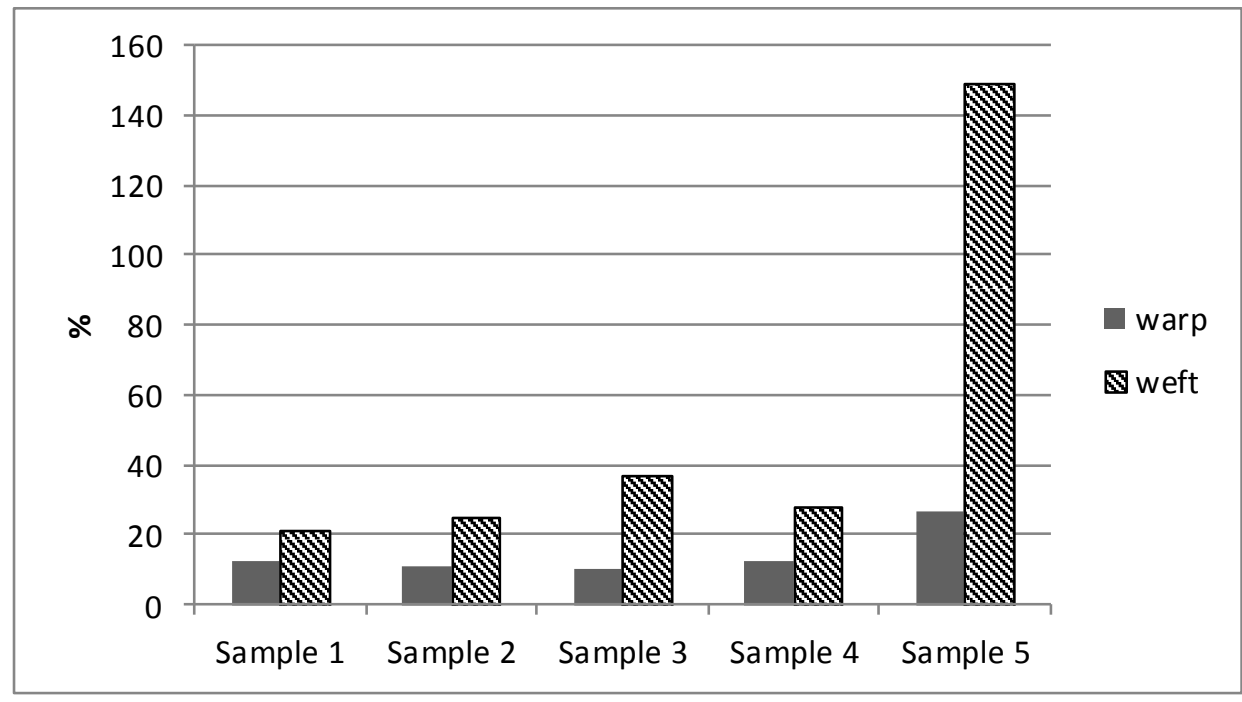

Figure 10. Elongation at break of investigated seersucker fabrics

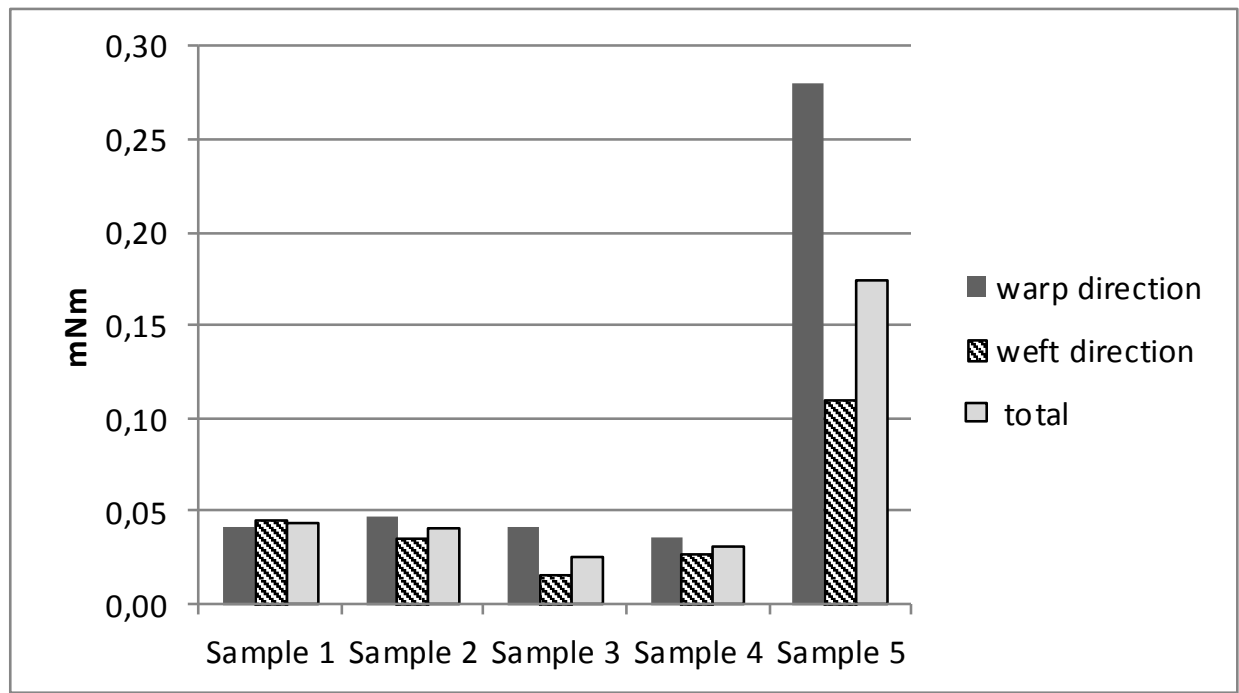

Figure 11. Bending stiffness of investigated seersucker fabrics

fabric is characterized by the lowest mass per square meter and thickness. All mentioned factors influenced the bending stiffness of the fabric.

\section{Conclusions}

The paper presents the investigations of the seersucker fabrics. Investigated fabrics were manufactured on the basis of the same warp set: basic warp and goffering warp, both made from the plied cotton $20 \times 2$ tex yarn. Different yarns were applied in weft. Performed investigations confirmed that a kind of weft yarn significantly influences the structural and mechanical properties of the seersucker fabrics. It was stated that the kind of weft yarn influences the properties of the seersucker fabrics in both warp and weft directions. Especially, an application of the elastomeric yarn in the weft caused the tightening of the structure of the fabric in weft direction resulting in radical changes of all structural and mechanical properties of fabric.
Presented investigations confirmed that by an appropriate selection of weft yarn, it is possible to shape the structure, appearance, and mechanical properties of the seersucker woven fabrics.

The performed investigations showed that the 3D laser scanning allows analyzing the surface topography of the seersucker fabrics. Results from the 3D laser scanning can be applied to calculate the total height of the profile $W_{z}$ of lines representing the fabric profile in place of cutting. Moreover, on the basis of the results from the $3 \mathrm{D}$ laser scanning, it is possible to assess the seersucker effect, with flat and puckered strips of the seersucker fabrics.

\section{References}

[1] MatusiakM., FrączakŁ. (2015). Investigation of Waviness of 3D Woven Fabrics. In: Innovations in Clothing Design, Materials, Technology and Measurement Methods, 
ed. By I. Frydrych, G. Bartkowiak, M. Pawłowa, Lodz University of Technology, ISBN 978-83-7283-666-3, 166-182

[2] Gandhi K. (2012). Woven Textiles Principles, Technologies and Applications, 1st ed., Woodhead Publishing, New Delhi, 142-158

[3] The American Heritage Dictionary of the English Language: Fifth Edition (2015). Available from: <https://ahdictionary. com > 02.03.2016

[4] Kyame G.J., Lofton J.T., Cool and Carefree Cotton Seersucker, Available from: <http://naldc.nal.usda.gov/ download/ IND43895201/PDF>

[5] Maqsood M., Nawab Y., Javaid M.U., Shaker K.,Umair M. (2014). Development of seersucker fabrics using single warp beam and modelling of their stretch-recovery behavior, The Journal of The Textile Institute, DOI 10.1081/00405000.2014.977542

[6] Matusiak M., Sikorski K., Wilk E. (2012). New Applications of Cotton Fibres, Fiber Society Spring Conference, St Gallen

[7] Ashraf W., Nawab Y., Maqsood M., Khan H., Awais H., Ahmad S., Ashraf M., Ahmad S. (2015). Development of
Seersucker Knitted Fabric for Better Comfort Properties and Aesthetic Appearance, Fibers and Polymers, 16(3), 699-701

[8] Willard D. (2011). The Fabric Selector, 1st ed., Search Press Ltd, 42-78

[9] Ghahraman F.G., Tavanai H., Hosseini S.A. (2010). A qualitative assessment of seersucker effect through spectral density and angular power spectrum function algorithms, The Journal of The Textile Institute, 101(3), 276-281

[10] Matusiak M., Frącczak Ł. (2016). Development of Functional 3D Woven Fabrics for Therapeutic Applications, Proceedings of The 90th Textile Institute World Conference, Poznan 2016.

[11] Militký J., Mazal M. (2007). Image analysis method of surface roughness evaluation, International Journal of Clothing Science and Technology, 19, 186-193

[12] Calvimontes A., Badrul Hasan M.M., Dutschk V. (2010). Effects of Topographic Structure on Wettability of Woven Fabrics. In: Woven Fabric Engineering, ed. by Dobnik Dubrovski P., ISBN 978-953-307-194-7, Publisher: Sciyo, 18, 71-92 